$04 ; 12$

\title{
Генерация быстрых заряженных частиц суперпозицией осциллирующих электрических полей со случайно прыгающими фазами
}

\author{
(C) В.М. Логинов
}

Красноярский государственный педагогический университет им. В.П. Астафьева

E-mail: valog_1949@mail.ru

Поступило в Редакцию 10 февраля 2017 г.

Исследовано движение нерелятивистской заряженной частицы в переменном электрическом поле, представляющем суперпозицию монохроматических волн, фазы которых являются скачкообразными случайными функциями времени. Статистический анализ проведен в рамках точно решаемой модели, где фазы считаются статистически независимыми случайными телеграфными сигналами. Вычислена средняя кинетическая энергия заряженной частицы. Показано, что есть множество значений характеристик случайных скачков фаз (их амплитуд и средних частот), при которых средняя энергия, осциллируя, растет со временем. На временах много больше характерных времен спада корреляций фаз средняя энергия растет линейно со временем (стохастический нагрев), при этом важно, что коэффициент роста немонотонно зависит от параметров скачков фаз и максимальный прирост пропорционален числу гармоник.

DOI: 10.21883/PJTF.2017.14.44819.16741

Как известно в стохастических электромагнитных полях может осуществляться эффективное ускорение заряженных частиц и нагрев плазмы [1,2]. В работах [3-7] экспериментально и компьютерным моделированием установлено, что микроволновое излучение со стохастически прыгающей фазой позволяет реализовать эффективный бесстолкновительный нагрев электронов и пробой газа при меньших напряженностях электрического поля, наблюдать эффект „просветления волновых барьеров“ и т.д. 
В данной работе использована модель случайных скачков фазы, позволяющая получить аналитические выражения для прироста средней кинетической энергии частицы.

Рассмотрим одномерное (вдоль оси $x$ ) движение нерелятивистской заряженной частицы в переменном электрическом поле, представленном суперпозицией $N$ плоских монохроматических электромагнитных волн со случайно прыгающими фазами $\alpha_{i}(t)$ :

$$
\frac{d V}{d t}=\sum_{i=1}^{N} \varepsilon_{i} \cos \left[\omega_{i} t+\alpha_{i}(t)\right]
$$

где $V$ - скорость частицы, $\varepsilon_{i}=q E_{i} / m, q, m$ - ее заряд и масса частицы, $E_{i}$ - амплитуда $i$-й гармоники с частотой $\omega$. В качестве моделей стохастически прыгающих фаз $\alpha_{i}(t)$ используются марковские дихотомические процессы (в отечественной литературе случайные телеграфные сигналы, см. [8]). Функции $\alpha_{i}(t)$ представляют собой ступенчатые случайные функции, принимающие с одинаковой вероятностью постоянные значения $\pm \sigma_{i}$. Функции имеют нулевое среднее $\left\langle\alpha_{i}(t)\right\rangle=0$ и экспоненциально спадающие корреляционные функции $K_{i}\left(\left|t_{1}-t_{2}\right|\right)=\left\langle\alpha_{i}\left(t_{1}\right) \alpha_{i}\left(t_{2}\right)\right\rangle=\sigma_{i}^{2} e^{-2 \lambda_{i}\left|t_{1}-t_{2}\right|}$. Угловые скобки означают усреднение по ансамблям реализаций процессов $\alpha_{i}(t)$. Здесь и далее процессы считаются статистически независимыми. Каждому процессу $\alpha_{i}(t)$ отвечает свое характерное время спада корреляций $\tau_{i}=1 / 2 \lambda_{i}$, а $\lambda_{i}$ - задает среднее число скачков в единицу времени процесса $\alpha_{i}(t)$. Для дихотомического шума $\alpha(t)$, принимающего значения $\pm \sigma$, справедливо равенство

$$
F(\alpha(t))=\frac{1}{2}[F(\sigma)+F(-\sigma)]+\frac{\alpha(t)}{2 \sigma}[F(\sigma)-F(-\sigma)]
$$

где $F(\alpha(t))$ - некоторая функция. Учитывая (2), уравнение (1) можно представить в виде

$$
\frac{d V}{d t}=\sum_{i=1}^{N} \varepsilon_{i}\left[\cos \sigma_{i} \cos \omega_{i} t-\frac{\sin \sigma}{\sigma_{i}} \alpha_{i}(t) \sin \omega_{i} t\right] .
$$

Уравнение (3) представляет собой исходное стохастическое дифференциальное уравнение.

Письма в ЖТФ, 2017, том 43, вып. 14 
Определим одноточечные моменты скорости частицы первого и второго порядка. Усредняя обе части (4) по ансамблям реализаций процессов $\alpha_{i}(t)$ и учитывая $\left\langle\alpha_{i}(t)\right\rangle=0$, получаем для средней скорости и координаты частицы

$$
\langle V(t)\rangle=\sum_{i=1}^{N} \varepsilon_{i} \frac{\sin \omega_{i} t}{\omega_{i}} \cos \sigma_{i}, \quad\langle X(t)\rangle=\sum_{i=1}^{N} \varepsilon_{i} \frac{\left(1-\cos \omega_{i} t\right)}{\omega_{i}^{2}} \cos \sigma_{i}
$$

Здесь принято $\langle V(0)\rangle=0$ и $\langle X(0)\rangle=0$. Вклад от взаимодействия частицы с суперпозицией плоских монохроматических волн со случайно прыгающими фазами для средней скорости и координаты проявляется в виде множителей $\cos \sigma_{i}$. Например, если на периоде $0 \leqslant \sigma_{i} \leqslant 2 \pi$ $\sigma_{i}=\pi / 2$ или $3 \pi / 2$, то частица в среднем локализуется в области начала координат.

Вычислим среднюю кинетическую энергию частицы. Для этого потребуется одноточечный момент $\left\langle V^{2}(t)\right\rangle$. Стохастическое уравнение для $V^{2}(t)$ имеет вид

$$
\frac{d V^{2}}{d t}=2 V \sum_{i=1}^{N} \varepsilon_{i}\left[\cos \sigma_{i} \cos \omega_{i} t-\frac{\sin \sigma_{i}}{\sigma_{i}} \alpha_{i}(t) \sin \omega_{i} t\right] .
$$

После усреднения по реализациям процессов $\alpha_{i}(t)$, получаем

$$
\frac{d\left\langle V^{2}\right\rangle}{d t}=2\langle V\rangle \sum_{i=1}^{N} \varepsilon_{i} \cos \sigma_{i} \cos \omega_{i} t-2 \sum_{i=1}^{N} \varepsilon_{i} \frac{\sin \sigma_{i}}{\sigma_{i}} \sin \omega_{i} t\left\langle\alpha_{i}(t) V\right\rangle .
$$

Среднее $\left\langle\alpha_{i}(t) V\right\rangle \equiv W_{i}$ преобразуем, используя метод формул дифференцирования (ФД) статистических средних [8]. Для дихотомического шума $\alpha(t)$ ФД имеет вид

$$
\frac{d}{d t}\left\langle\alpha(t) \Phi_{l}[\alpha]\right\rangle=-v\left\langle\alpha(t) \Phi_{l}[\alpha]\right\rangle+\left\langle\alpha(t) \frac{d \Phi_{l}[\alpha]}{d t}\right\rangle,
$$

где $\Phi_{l}[\alpha]=\Phi_{l}\left[\alpha\left(t^{\prime}\right)\right]$ с $t^{\prime} \leqslant t-$ запаздывающий функционал процесса $\alpha(t)$. Здесь введено обозначение $v=2 \lambda$. Полагая в (7) $\Phi_{l}[\alpha]=V(t)$ и используя для преобразования производной $d V / d t$ стохастическое уравнение движения (3), получаем замкнутую систему уравнений для

Письма в ЖТФ, 2017, том 43, вып. 14 
определения средних $\left\langle V^{2}\right\rangle$ и $W_{i}$ (здесь учтено, что $\alpha_{i}^{2}(t) \equiv \sigma_{i}^{2}=\mathrm{const}$ для любых $i=1,2, \ldots, N)$

$$
\begin{gathered}
\frac{d\left\langle V^{2}\right\rangle}{d t}=2\langle V\rangle \sum_{i=1}^{N} \varepsilon_{i} \cos \sigma_{i} \cos \omega_{i} t-2 \sum_{i=1}^{N} \varepsilon_{i} \frac{\sin \sigma_{i}}{\sigma_{i}} W_{i} \sin \omega_{i} t \\
\frac{d W_{i}}{d t}=-v_{i} W_{i}-\varepsilon_{i} \sigma_{i} \sin \sigma_{i} \sin \omega_{i} t .
\end{gathered}
$$

При выводе уравнения (8) учтено, что процессы $\alpha_{i}(t)$ и $\alpha_{i}(t)$ статистически независимы, так что $\left\langle\alpha_{i}(t) \alpha_{j}(t)\right\rangle=\delta_{i j} \sigma_{i}^{2}$. Уравнения (8), (9) решаем при нулевых начальных условиях. В результате для дисперсии скорости заряженной частицы $\Delta_{V}(t)=\left\langle V^{2}(t)\right\rangle-\langle V(t)\rangle^{2}$ в модели электрического поля в виде суперпозиции гармоник со случайно прыгающими фазами получаем

$$
\Delta_{V}(t)=\sum_{i=1}^{N} \varepsilon_{i}^{2} \frac{\sin ^{2} \sigma_{i}}{v_{i}^{2}+\omega_{i}^{2}}\left(p\left(v_{i}, \omega_{i}, t\right)+q\left(v_{i}, \omega_{i}, t\right)\right),
$$

где

$$
\begin{gathered}
p\left(v_{i}, \omega_{i}, t\right)=v_{i} t+\frac{1}{2}\left(\cos 2 \omega t-\frac{v_{i}}{\omega_{i}} \sin 2 \omega_{i} t-1\right) \\
q\left(v_{i}, \omega_{i}, t\right)=\frac{2 \omega_{i}^{2}}{v_{i}^{2}+\omega_{i}^{2}}\left[1-\left(\frac{v_{i}}{\omega_{i}} \sin \omega_{i} t+\cos \omega_{i} t\right) e^{-v_{i} l}\right] .
\end{gathered}
$$

Обращает на себя внимание, что зависимость от амплитуд прыгающих фаз в динамике моментов скорости частицы различна. В выражение для средней скорости входят косинусы фаз, а среднеквадратичные флуктуации скорости содержат синусы фаз. В частности, это означает, что при $\sigma_{i}=\pi n_{i}, n_{i}=0,1,2, \ldots$ дисперсия скорости $\Delta_{V}(t)$ равна нулю и $\left\langle V^{2}(t)\right\rangle \equiv\langle V(t)\rangle^{2}$. Прирост средней кинетической энергии частицы за счет случайных флуктуаций фаз определяется квадратом средней скорости, так что справедлива оценка $\left\langle V^{2}(t)\right\rangle \leqslant \sum_{i, j=1}^{N}\left|\varepsilon_{i}\right|\left|\varepsilon_{j}\right| \frac{1}{\omega_{i} \omega_{j}}$. При $\sigma_{i} \neq \pi n_{i}$ максимальный вклад в дисперсию, а соответственно и в прирост средней кинетической энергии частицы от амплитуды сдвига, единица (при этом $\cos \sigma_{i}=0$ и $\left.\langle V(t)\rangle=0,\langle X(t)\rangle=0\right)$.

Письма в ЖТФ, 2017, том 43, вып. 14 
Интересуясь приростом средней кинетической энергии частицы за счет взаимодействия с флуктуирующим полем, рассмотрим асимптотику $t \gg \tau=\max \left(1 / \nu_{i}\right)$ (на временах много больше характерного времени спада корреляций). В этом случае

$$
\Delta_{V}(t)=\left(\sum_{i=1}^{N} \varepsilon_{i}^{2} \frac{\sin ^{2} \sigma_{i}}{v_{i}^{2}+\omega_{i}^{2}} v_{i}\right) t .
$$

Средняя кинетическая энергия линейно растет со временем (стохастический нагрев), причем важно, что скорость роста определяется суммой $N$ величин, которые пропорциональны квадратам амплитуд $E_{i}$ электрического поля (входят в $\varepsilon_{i}^{2}$ ), и произведением двух немонотонных функций, зависящих от характеристик случайных скачков фаз частот $v_{i}$ и амплитуд сдвига $\sigma_{i}$.

Функции $\sin ^{2} \sigma_{i}$ на периоде $0 \leqslant \sigma_{i} \leqslant 2 \pi$ в точках $\pi / 2$ и $3 \pi / 2$ принимают максимальные значения, равные 1. Функции $v_{i} /\left(v_{i}^{2}+\omega_{i}^{2}\right)$ принимают максимальные значения при $v_{i}=\omega_{i}$, т. е. когда частота скачков $v_{i}$ случайной фазы $i$-волны совпадает с частотой ее осцилляций $\omega_{i}$. Окончательно максимальный прирост средней энергии заряженной частицы за время определяется выражением

$$
\max \Delta_{V}(t)=\left(\sum_{i=1}^{N} \varepsilon_{i}^{2} \frac{1}{2 \omega_{i}}\right) t .
$$

Примем, что все слагаемые $\varepsilon_{i}^{2} / \omega_{i} \leqslant a$, тогда оценка максимального прироста энергии частицы принимает вид $\max \Delta_{l}(t) \leqslant \frac{1}{2} a N t$, т.е. величина прироста средней энергии увеличивается (пропорционально) за счет увеличения числа гармоник поля. В обзоре [9] отмечается большой интерес к новым методам ускорения частиц, основанный на синхронной работе большого числа волоконных лазеров с высокой средней мощностью.

Рассмотренная модель стохастических скачков фаз волн позволила аналитически проанализировать первые и вторые моменты скорости заряженной частицы. Показано, что статистические характеристики движения частицы зависят от амплитуд и средних частот скачков случайных фаз. Максимальный эффект стохастического нагрева проявляется, если $\sin \sigma_{i} \neq 0, i=1, \ldots, N$. На временах много больше времени спада корреляций фаз средняя кинетическая энергия частицы линейно растет со временем, с коэффициентом роста в виде суммы неотрицательных немонотонных от параметров фаз слагаемых.

Письма в ЖТФ, 2017, том 43, вып. 14 


\section{Список литературы}

[1] Файнберг Я.Б. // Физика плазмы. 2000. Т. 26. № 4. С. 362.

[2] Файнберг Я.Б., Басс Ф.Г., Шапиро В.Д. // ЖЭТФ. 1965. Т. 49. № 1 (7). С. 329.

[3] Карась В.И., Файнберг Я.Б., Алисов А.Ф. и др. // Физика плазмы. 2005. Т. 31. № 9. C. 810 .

[4] Карась В.И., Алисов А.Ф., Артамошкин А.М. и др. // Вопросы атомной науки и техники. Сер. Плазменная электроника и новые методы ускорения. 2006. № 5. C. 54 .

[5] Андреев Д.Г., Ерохин Н.С. // Прикладная физика. 2012. № 2. С. 5.

[6] Буи, В.А., Кузьмин В.В., Толстолужсский А.П. // Вопросы атомной науки и техники. Физика плазмы. 2013. № 1 (83). С. 137.

[7] Андреев Д.Г., Ерохин Н.С., Зольникова Н.Н. // Физика плазмы. 2012. Т. 38. № 8. C. 716.

[8] Шапиро В.Е., Логинов В.М. Динамические системы при случайных воздействиях. Новосибирск: Наука, 1983.

[9] Костюков И.Ю., Пухов А.М. // УФН. 2015. Т. 185. № 1. С. 89. 